Enserink, R., Ypma, R., Donker, G.A., Smit, H.A., Pelt, W. van. Infectious disease burden related to child day care in the Netherlands. Pediatric Infectious Disease Journal: 2013, 32(8), e334e340

\begin{tabular}{|l|l|}
\hline $\begin{array}{l}\text { Postprint } \\
\text { Version }\end{array}$ & 1.0 \\
\hline Journal website & $\underline{\text { http://dx.doi.org/10.1097/INF.0b013e318290601e }}$ \\
\hline Pubmed link & $\underline{\text { http://www.ncbi.nlm.nih.gov/pubmed/23584578 }}$ \\
\hline DOI & $10.1097 /$ INF.0b013e318290601e \\
\hline
\end{tabular}

This is a NIVEL certified Post Print, more info at http://www.nivel.eu

\title{
Infectious Disease Burden Related to Child Day Care in The Netherlands
}

\author{
REMKO ENSERINK ${ }^{1,2}$, MSC, ROLF YPMA, MSC ${ }^{1,2}$, GÉ A. DONKER, MD ${ }^{3}$, HENRIETTE A. SMIT, \\ $\mathrm{PHD}^{2}$, AND WILFRID VAN PELT, PHD ${ }^{1}$ \\ ${ }^{1}$ Center for Infectious Disease Control (Epidemiology and Surveillance Unit), National \\ Institute for Public Health and the Environment (RIVM), Bilthoven, The Netherlands; \\ ${ }^{2}$ Julius Center for Health Sciences and Primary Care, University Medical Center Utrecht, \\ Utrecht, The Netherlands; \\ ${ }^{3}$ NIVEL, Netherlands Institute of Health Services Research, Utrecht, The Netherlands
}

\begin{abstract}
Background: Studying day care-associated infectious disease dynamics aids in formulating evidence-based guidelines for disease control, thereby supporting day care centers in their continuous efforts to provide their child population with a safe and hygienic environment. The objective of this study was to estimate the (excess )infectious disease burden related to child day care attendance in the Netherlands.

Methods: A Dutch surveillance network of child day care centers (DCCs) prospectively reported on infectious disease episodes and related use of health care among their child population on a daily basis from March 2010 - March 2012.

Results: Gastroenteritis (387 per 1000 child-years) and influenza-like illness (247 per 1000 child-years) were the most frequently reported infectious diseases. DCCs reported these infectious diseases to occur twice as often among children aged 0-2 years old compared to children aged 2-4 years old. Antibiotic treatment was required in 6\%, a general practitioner visit in $29 \%$ and hospitalization in $2 \%$ of infectious disease episodes. DCC incidences of gastroenteritis and influenza-like illness requiring children to visit a general practitioner were approximately twice as high as general population estimates for this age group. Part of the DCCs indicated to not always wash the hands of children before eating (34\%) or after a toilet visit (15\%) or to not always clean the toilet and kitchen areas $(17 \%)$ on a daily basis. Conclusion: The infectious disease risk associated with child day care attendance is substantial, particularly among the very young attendees, in excess of general population estimates for this age group and potentially partly preventable.
\end{abstract}

\section{INTRODUCTION}

In the Netherlands, approximately $50 \%$ of the 0.7 million Dutch children aged 0 to 4 years are cared for in child day care centers (DCCs). DCCs provide care for an immunological immature, dynamic and crowded population and therefore represent a setting conductive to transmission of infectious diseases. As a result, children cared for at DCCs are at an increased risk of acquiring infectious diseases ${ }^{1,2,3}$. The 
Enserink, R., Ypma, R., Donker, G.A., Smit, H.A., Pelt, W. van. Infectious disease burden related to child day care in the Netherlands. Pediatric Infectious Disease Journal: 2013, 32(8), e334e340

increased risk has direct impact on the child as it leads to infectious disease episodes that are greater innumber ${ }^{4}$, especially in young children with underlying disease ${ }^{5}$. However, the increased occurrence also indirectly affects society as attending children readily transmit DCC-related infectious diseases and possible related antimicrobial resistance ${ }^{6}$ from the DCC environment via caretakers ${ }^{7}$, parents and family ${ }^{8}$ into the society at large. These transmission events have social and economic consequences as they result in increased work absenteeism for parents and increased expenses on medical care ${ }^{9}$.

The impact of DCC-related infectious diseases is widely recognized as being substantial and numerous guidelines exist for their control ${ }^{10,11,12}$. However, our knowledge and understanding of the disease burden is hampered by lack of dedicated surveillance in the child day care setting. At least in the Netherlands, surveillance is restricted to short-term research initiatives ${ }^{13,14}$, a mandatory infectious disease notifiable reporting systems and a voluntary physician reporting network ${ }^{15}$. Notifiable reporting systems are often locally organized and monitor outbreaks only and the physician network does not discriminate between visiting children that do and do not attend day care. In addition, both systems largely underestimate true infectious disease incidence; by far the majority of (DCC-related) infectious diseases are sporadic and mild in nature and will therefore not present themselves to these systems. As a result, these research initiatives and reporting systems are designed neither to quantify the infectious disease burden related to child day care attendance nor to relate infectious disease incidence to risk factors present in the DCC setting. Accurate incidence estimates provide a first step towards identification of effective infectious disease control measures and public health priority setting that, ultimately, will lead to improvement of the health and well-being of day care-attending children. Since 2010, the Dutch Institute for Public Health therefore implements an ongoing national surveillance network (acronym: KIzSS) of DCCs ${ }^{16}$. We are unaware of any other national initiative that systematically collects data on the infectious disease occurrence in child day care.

This paper has two objectives. (1): Estimate the incidence of common childhood infectious disease episodes and related antibiotics consumption, general practitioner visits and hospitalization among Dutch day care attendees and (2): Estimate the excess incidence of two frequent infectious diseases - gastroenteritis and influenzalike illness- attributable to Dutch day care attendance ${ }^{17}$.

\section{MATERIALS AND METHODS}

\section{Day-Care Center Recruitment}

All DCCs registered in a database provided by the ministry of Education, Culture and Sciences $(n=3913)$ were invited in November 2009 to participate in a crosssectional questionnaire survey into sociodemographics, facility setup, and infectious disease policies.

DCCs from areas of all degrees of urbanization were equally represented and were comparable with regard to socioeconomic classification. As part of the questionnaire survey, DCCs were invited to participate in the surveillance network. DCCs not willing to take part in the questionnaire survey were asked to answer a short nonresponse questionnaire. DCCs interested in participation were visited by the research team and included in the study. For a detailed description of the design, 
Enserink, R., Ypma, R., Donker, G.A., Smit, H.A., Pelt, W. van. Infectious disease burden related to child day care in the Netherlands. Pediatric Infectious Disease Journal: 2013, 32(8), e334= e340

methodology and definitions on infectious diseases implemented by the surveillance network study we refer to our study design article published elsewhere ${ }^{16}$.

\section{Data collection}

The KIzSS network has a prospective cohort design, following day care centers rather than individual children over time. DCCs participating in the surveillance network used study-provided calendars to indicate the daily occurrence of predefined infectious disease episodes (table 1) among their child-population aged 0-4 years. Day care centers only reported infectious disease episodes that resulted in absence of the child on one or more days that the child was scheduled to attend day care. Children were called in sick by their parent(s) or were sent home sent by the DCC staff due to illness. Per reporting, additional information regarding related health-care-utilization characteristics was provided including possible antibiotic consumptions, general practitioner visits and hospitalizations. A child had to be fully recovered for at least 7 days before being reported ill again. In addition, DCCs reported the number of children enrolled for day care per week of participation. Both incidence numerators and denominators were reported by DCCs via a webbased registration tool ${ }^{18}$ on a daily basis to the Dutch Institute of Public Health. A researcher checked all reports for completeness. Reports that were unclear or incomplete were sent back via the digital reporting system to the DCC with the request to make appropriate changes before finalizing the report. DCCs that did not report at least one incidence numerator/denominator per month for a minimal period of 6 months during the study period and duplicate reports were excluded from further analysis.

In addition, the Dutch Sentinel General Practice (GP) Network provided national estimates of general practitioner visits for GE and ILI between March 2010 and March 2011.

Similar data for the other infectious diseases measured in KIzSS or for the year March 2011 - March 2012 were not (yet) available. In the GP network, sentinel doctors were asked to report patients fulfilling the definitions of GE or ILI (table 1). For a detailed description of the design, methodology and definitions implemented by the GP network we refer to an article published elsewhere ${ }^{17}$.

\section{STATISTICAL ANALYSIS}

\section{Definitions and assumptions}

An infectious disease episode, the incidence numerator, was defined as a child experiencing one or more days of pre-defined illness that results in one or more days of absence from day care. The child-time at risk, the incidence denominator, was defined as the number of children enrolled for day care per week of participation in the surveillance network. As children do not attend day care every day of the week, we adjusted for episodes missed due to children getting ill and recovering in between DCC attendance days. Infectious disease incidence was therefore calculated as the number of infectious disease episodes per 1000 child-years at risk adjusted by an infectious disease dependent correction factor for illness episodes missed (Appendix I, available on request to the authors). Weeks in which a DCC reported the number of children attending that week, but did not report infectious disease episodes during that week, were considered to be free from absence due to infectious disease during that week. The relative difference in infectious disease incidence between children of 
Enserink, R., Ypma, R., Donker, G.A., Smit, H.A., Pelt, W. van. Infectious disease burden related to child day care in the Netherlands. Pediatric Infectious Disease Journal: 2013, 32(8), e334e340

different age (0-2 year olds compared to 2-4 year olds), study year (2010-2011 compared to 2011-2012) and population (day care-based surveillance compared to GP based surveillance) were calculated and expressed in an Incidence Rate Ratio (IRR). The association between an infectious disease and age were based on DCC based age-stratified denominator information of their child population.

\section{Representativeness of the surveillance network}

The representativeness and diversity of the DCC surveillance network was assessed by comparing the DCC characteristics socioeconomic classification, degree of urbanization, facility design, and hygiene practices between network participants and non-participants. Differences at the level of a p-value $<=0.05$ were considered statistically significant. In addition, a principal component analysis was performed to assess whether the overall variation in combinations of DCC characteristics in participants reflected the overall variation among all DCCs (Appendix II, available on request to the authors). Data were analyzed using the statistical software package STATA/SE 11.2 for Windows.

\section{Ethical and funding considerations}

This study is conducted according to the principles of the Declaration of Helsinki. The Dutch Central Committee on Research involving Human Subjects in Utrecht, The Netherlands, endorsed ethical approval to conduct this study. No subjectidentifiable results are generated.

This paper presents independent research commissioned by the Dutch National Institute for Public Health and the Environment and has been internally financed.

\section{RESULTS}

\section{The Surveillance Network}

Figure 1 depicts the dynamics of the day care center network during the study period. We approached 3913 DCCs. Of the approached DCCs, 2612 DCCs (67\%) did not respond at all and 356 DCCs (9\%) indicated that they lacked time and/or interest to participate in either the survey among DCCs or the surveillance network. 945DCCs (24\%) did respond by returning a completed questionnaire regarding their baseline characteristics. Of these DCCs, 19 (2\%) were enrolled in the KIzSS cohort in March 2010. Additionally, selective recruitment was performed during the study period to ensure regional representativeness, resulting in additional inflow of 42 DCCs during the study period. Similarly, there was an outflow of 36 DCCs ending their cohort participation efforts during the study period. At the end of the considered study period in March 2012, the surveillance network counted 25 participating DCCs. In total, 40 DCCs participated for at least 26 weeks (10\%, 50\% and 90\% percentiles: 33, 49 and 101participation-weeks respectively) between March 2010 to March 2012.

\section{Baseline characteristics of DCCs in the surveillance network}

Table 2 displays a selection of several important baseline characteristics of DCCs that participated in the questionnaire survey and of potential differences between DCCs that did $(\mathrm{N}=40)$ and did not $(\mathrm{N}=905)$ participate in the DCC cohort during the study period. A detailed overview of DCC characteristics and their interrelatedness is available on request to the authors (Appendix II). On average, a DCC enrolled 82 children. The child/caregiver ratio increased with decreasing DCC capacity. On 
Enserink, R., Ypma, R., Donker, G.A., Smit, H.A., Pelt, W. van. Infectious disease burden related to child day care in the Netherlands. Pediatric Infectious Disease Journal: 2013, 32(8), e334= e340

average, a DCC staff member was responsible for the care of 5 children. DCCs were organized either horizontally (children of similar age are grouped together), vertically (children of all ages are grouped together) or both. Small DCCs had a tendency to be organized vertically, whereas larger DCCs were more often organized horizontally. Smaller DCCs located in rural settings applied less staff mixing between groups, higher cleaning frequencies of toilet and kitchen facilities and higher consistency in implementation of exclusion policy guidelines compared to larger DCCs.

Part of the of DCCs indicated they did not always wash the hands of children before eating (34\%) or after a toilet visit (15\%) or they did not always clean the toilet and kitchen areas (17\%) on a daily basis despite their written policies to do so. $42 \%$ of DCCs did not have a dedicated diaper changing station. Cohorting or exclusion of children with GE or ILI was not strictly part of DCC infectious disease control policy, regardless of whether an infectious disease outbreak was suspected or not. Rather, DCCs indicated that they excluded children too ill to participate in daily DCC activities. Half of the DCCs contacted public health authorities in case of a suspected outbreak that is a notifiable event.

Between DCCs participating in our surveillance network and all DCCs that responded to our questionnaire, no significant differences were detected regarding the distribution of socioeconomic status and degree of urbanization between DCC responders and non-responders and no significant differences were detected in terms of organization, facility design and hygiene policies. The representativeness of the DCC surveillance network was further illustrated in a principal component analysis on the DCC profile of combinations of these DCC characteristics, showing that the variation of KIzSS cohort participants reflected the national variation in DCC characteristics very well (Appendix II, available on request to the authors).

\section{Infectious disease incidence and related health care utilization}

Figure 2A, 2B and 2C show the monthly infectious disease incidence of gastrointestinal, respiratory and exanthematous disease respectively as measured in the KIzSS network between March 2010 and March 2012. Table 3 presents the number of infectious disease episodes per 1000 child-years between March 2010 and March 2012, stratified by study year and child's age.

Per infectious disease the fraction of children requiring antibiotics, a general practitioner visit and hospitalization are given. Finally, table 4 depicts difference in GE and ILI incidence requiring a visit to the general practitioner between the DCCbased and the GP-based surveillance during the period March 2010- March 2011.

\section{Incidence}

A total of 2488 infectious disease episodes leading to absence from day care were estimated to have occurred during 2441 child-years of follow-up; approximately one episode with absence per child-year. The highest infectious disease incidence with absence was observed for GE (387) and ILI (247). Ear infection (150), chickenpox (120), exanthema (59), eye infection (39) and impetigo (17) were reported less frequently.GE and ILI displayed clear seasonality with a distinct 4 fold increase in incidence during the winter months. No regular time-related incidence patterns were observed for other infectious diseases, although chickenpox incidence peaked with some regularity during the study because of individual/single DCC outbreaks. 
Enserink, R., Ypma, R., Donker, G.A., Smit, H.A., Pelt, W. van. Infectious disease burden related to child day care in the Netherlands. Pediatric Infectious Disease Journal: 2013, 32(8), e334= e340

The incidence of all infectious diseases reported was significantly lower in 2010/2011 compared to 2011/2012 with the exception of chickenpox (significantly higher) and exanthema (no difference). Compared to 2-4 year old children, 0-2 year old children were at a 1.2 (chicken pox) to 3.6 (eye infection) times significantly higher risk for infectious diseases monitored, with the exception of impetigo ( 0.5 times significantly lower risk). Overall, children aged 0-2 years were approximately at a two times higher risk of experiencing an infectious disease episode compared to 2-4 year old children.

Overall, children received antibiotic treatment in6\% of infectious disease episodes, $29 \%$ consulted a GP and 2\% needed to be hospitalized. Antibiotics were most often prescribed for impetigo (24\%), ear infections (21\%) and ILI (8\%). Impetigo and ear infections were also the most common reason for a child to visit the general practitioner: $93 \%$ and $62 \%$ respectively. The highest risks for hospitalization were reported for episodes of ear infection (4.4\%), ILI (2.3\%) and GE (1.6\%). Children attending a DCC consulted their GP more often for GE and ILIcompared to children in the Dutch Sentinel GP Network of the same age group and during the same period (IRRGE: 1.7 [1.4-2.0], IRR ILI: 1.8 [1.6-2.1]). In case of ILI, this effect was even stronger for the $0-2$ year olds (IRR: 2.3 [1.9-2.7]).

\section{DISCUSSION}

Gastroenteritis (GE) and influenza-like (ILI) illness were the most commonly reported infectious diseases among children in our day care-based surveillance network, particularly during the winter months. Children experiencing these infectious diseases consulted the GP approximately two times as often as reported by the Dutch Sentinel GP Network during the same period and for the same age group. For ILI, this is especially true among the very young, suggesting that early daycare attendance shifts the occurrence of first infectious disease episodes to an earlier age where complications requiring health care are more likely to arise. Qualitatively, our results are in agreement with those reported in other studies ${ }^{19,20}$. However, we have three reasons to believe that we underestimated the true disease burden related to day care attendance. Firstly, DCCs only included illness episodes that required a child to be sent or kept home, whereas other studies have shown that only $50 \%$ of children might require home-care due to illness ${ }^{21}$. Indeed, the GE incidence measured in our DCC surveillance network was lower than estimated in a community-based study that was performed among the same age group in the Netherlands in $1999^{13}$ using diaries. Secondly, the presented GP-based incidence estimates for GE and ILI were calculated based on a at risk child population of which $50 \%$ regularly attends day care. Thirdly, both DCCs as well as GP's may not have reported all eligible disease episodes among their populations.

We have shown the importance of including multiple surveillance years as infectious disease incidences might vary significantly over the years. There is a possibility that the disease burden related to GE and ILI measured during the study period differ from the disease burden of other years. Although the DCC network is representative with regard to distributions in the degree of urbanization and the socioeconomic status of DCCs that did and did not respond to our questionnaire survey, some differences may exist in other DCC characteristics for which we had no information. It is expected that these will be of minor importance for the estimates, rank order and relative importance of the infectious diseases presented here. 
Enserink, R., Ypma, R., Donker, G.A., Smit, H.A., Pelt, W. van. Infectious disease burden related to child day care in the Netherlands. Pediatric Infectious Disease Journal: 2013, 32(8), e334 e340

Results from our DCC survey indicate that there is ample room for improvement in implementing simple measures for infectious disease prevention such as hand hygiene and appropriate cleaning of contaminated surfaces. Such measures have convincingly shown to reduce the infectious disease risk in the DCC setting ${ }^{22,23,24}$; especially against enteric infections ${ }^{25}$ which were most commonly reported during our study. It is not clear whether the performance of Dutch DCCs lag behind internationally recognized standards as was the case in $2005^{26}$.

The national baseline estimates of infectious disease incidences presented in this paper will provide a basis for future disease burden, cost of illness, risk factor and intervention studies.

Together, these studies will identify most efficient hygiene standards and control measures in practice in DCCs in the Netherlands and give justification for their implementation. This may convince and stimulate DCCs in fulfilling their responsibility to provide children with a save and hygienic environment as is required of DCCs since 2005 by Dutch DCC law. The KIzSS surveillance network will continue to monitor child day care-associated infectious disease dynamics in the future.

\section{REFERENCES}

1. Nesti MMM, Goldbaum M. Infectious diseases and daycare and preschool education. Jornal de Pediatria. 2007;83(4):299-312.

2. Development $\mathrm{H}$, Child $\mathrm{E}$. Child care and common communicable illnesses: results from the National Institute of Child Health and Human Development Study of Early Child Care. Archives of pediatrics \& adolescent medicine. 2001;155(4):481-8.

3. Brady MT. Infectious disease in pediatric out-of-home child care. American journal of infection control. 2005;33(5):276-85.

4. Bradley RH. Child care and common communicable illnesses in children aged 37 to 54 months. Archives of pediatrics \& adolescent medicine. 2003;157(2):196-200.

5. McGrath-Morrow S a, Lee G, Stewart BH, et al. Day care increases the risk of respiratory morbidity in chronic lung disease of prematurity. Pediatrics. 2010;126(4):632-7.

6. Dunais B, Pradier C, Carsenti $\mathrm{H}$, et al. Influence of child care on nasopharyngeal carriage of Streptococcus pneumoniae and Haemophilus influenzae. The Pediatric infectious disease journal. 2003;22(7):589-92.

7. Marshall BC, Adler SP. The frequency of pregnancy and exposure to cytomegalovirus infections among women with a young child in day care. American journal of obstetrics and gynecology. 2009;200(2):163.e1-5.

8. Fau C, Billaud G, Pinchinat S, et al. [Epidemiology and burden of rotavirus diarrhea in day care centers in Lyon, France]. Archives de pédiatriel: organe officiel de la Sociéte française de pédiatrie. 2008;15(7):1183-92.

9. Van der Wielen M, Giaquinto C, Gothefors L, et al. Impact of community-acquired paediatric rotavirus gastroenteritis on family life: data from the REVEAL study. BMC family practice. 2010;11:22.

10. Pediatrics AA of. Caring for our children: Guidelines for Out-of-Home Child Care.; 2002.

11. American Academy of Pediatrics. Red Book: 2012 Report of the Committee on Infectious Diseases.; 2012.

12. American Academy of Pediatrics. Managing Infectious Diseases in Child Care and Schools: A Quick Reference Guide, 2nd Edition.

13. De Wit MA, Koopmans MP, Kortbeek LM, et al. Sensor, a population-based cohort study on gastroenteritis in the Netherlands: incidence and etiology. American journal of epidemiology. 2001;154(7):666-74.

14. De Wit M a, Koopmans MP, Kortbeek LM, Van Leeuwen NJ, Bartelds Al, Van Duynhoven YT. Gastroenteritis in sentinel general practices, The Netherlands. Emerging infectious diseases. 2001;7(1):82-91. 
Enserink, R., Ypma, R., Donker, G.A., Smit, H.A., Pelt, W. van. Infectious disease burden related to child day care in the Netherlands. Pediatric Infectious Disease Journal: 2013, 32(8), e334 e340

15. Dijkstra F, Donker G a, Wilbrink B, Van Gageldonk-Lafeber a B, Van Der Sande M a B. Long time trends in influenza-like illness and associated determinants in The Netherlands. Epidemiology and infection. 2009;137(4):473-9.

16. Enserink R, Noel H, Friesema I, et al. The KIzSS network, a sentinel surveillance system for infectious diseases in day care centers: study protocol. BMC infectious diseases. 2012;12(1):259.

17. Donker GA. Continuous Morbidity Registration Dutch Sentinel General Practice Network 2011. Annual report. Utrecht, NIVEL, 2012. www.nivel.nl/peilstations.

18. Ward M, Brandsema $P$, Van Straten E, Bosman A. Electronic reporting improves timeliness and completeness of infectious disease notification, The Netherlands, 2003. Euro surveillanceı: bulletin européen sur les maladies transmissibles = European communicable disease bulletin. 2005;10(1):27-30.

19. Lu N, Samuels ME, Shi L, Baker SL, Glover SH, Sanders JM. Child day care risks of common infectious diseases revisited. Child: care, health and development. 2004; 30(4): 361-8.

20. Côté SM, Petitclerc A, Raynault M-F, et al. Short- and long-term risk of infections as a function of group child care attendance: an 8-year population-based study. Archives of pediatrics \& adolescent medicine. 2010;164(12):1132-7.

21. Slack-Smith LM, Read a W, Stanley FJ. Absence from childcare for respiratory illness. Child: care, health and development. 2004;30(1):29-37.

22. Luby SP, Curtis V. Hand washing for preventing diarrhoea. International journal of epidemiology. 2008;37(3):470-3.

23. Kotch JB, Isbell $P$, Weber DJ, et al. Hand-washing and diapering equipment reduces disease among children in out-of-home child care centers. Pediatrics. 2007;120(1):e29-36

24. Lennell A, Kühlmann-Berenzon S, Geli $P$, et al. Alcohol-based hand-disinfection reduced children's absence from Swedish day care centers. Acta paediatrica (Oslo, Norwayı: 1992). 2008;97(12):1672-80.

25. Roberts L, Jorm L, Patel M, Smith W, Douglas RM, McGilchrist C. Effect of infection control measures on the frequency of diarrheal episodes in child care: a randomized, controlled trial. Pediatrics. 2000;105(4 Pt 1):743-6.

26. Vermeer HJ, Van ljzendoorn MH, De Kruif REL, et al. Child care in The Netherlands: trends in quality over the years 1995-2005. The Journal of genetic psychology. 2008;169(4):360-85.

\section{TABLES AND FIGURES}

\begin{tabular}{|c|c|c|}
\hline $\begin{array}{l}\text { Infectious } \\
\text { Disease }\end{array}$ & KIzSS Network Definition & GP Network Definition \\
\hline GE & $\begin{array}{l}\text { Sudden, nonchronic, onset of } \geq 3 \text { episodes of watery } \\
\text { stools per day and/or sudden, nonchronic, onset } \\
\text { of } \geq 3 \text { emetic (vomiting) episodes per day. }\end{array}$ & $\begin{array}{l}\text { Three or more loose stools per day or diarrhea with } \geq 2 \\
\text { additional symptoms or vomiting with } \geq 2 \text { additional } \\
\text { symptoms. Additional symptoms included abdomi- } \\
\text { nal pain, abdominal cramps, nausea, fever, blood in } \\
\text { the stool, mucus in the stool, diarrhea or vomiting. }\end{array}$ \\
\hline ILI & $\begin{array}{l}\text { Acute or sudden onset of symptoms with fever }\left(\geq 38^{\circ} \mathrm{C}\right) \\
\text { and/or warm to the touch with suspicion of fever } \\
\text { and } \geq 1 \text { of the following symptoms: cough, rhinitis, } \\
\text { sore throat, listlessness, shortness of breath. }\end{array}$ & $\begin{array}{l}\text { Acute or sudden onset of symptoms with fever }\left(238^{\circ} \mathrm{C}\right) \\
\text { and } \geq 1 \text { of the following symptoms: cough rhinitis, } \\
\text { sore throat, frontal headache, retrosternal pain or } \\
\text { myalgia. }\end{array}$ \\
\hline Ear infection & Ear ache and/or runny ear with fever and/or common cold. & \\
\hline Chicken pox & $\begin{array}{l}\text { Sudden rash of small red bumps, followed by itchy } \\
\text { blisters and crust. }\end{array}$ & \\
\hline Exanthema & Spots on skin, rash. & \\
\hline Eye infection & $\begin{array}{l}\text { Red eyes and swollen eyelids with or without } \\
\text { yellow/green mucal discharge from eye. }\end{array}$ & \\
\hline Impetigo & $\begin{array}{l}\text { Expanding small red papules followed by a } \\
\text { honey-colored crust. }\end{array}$ & \\
\hline
\end{tabular}

FIGURE 1. Recruitment and inclusion dynamics of the DCC surveillance network from March 2010 to March 2012 in the Netherlands. 
Enserink, R., Ypma, R., Donker, G.A., Smit, H.A., Pelt, W. van. Infectious disease burden related to child day care in the Netherlands. Pediatric Infectious Disease Journal: 2013, 32(8), e334 e340

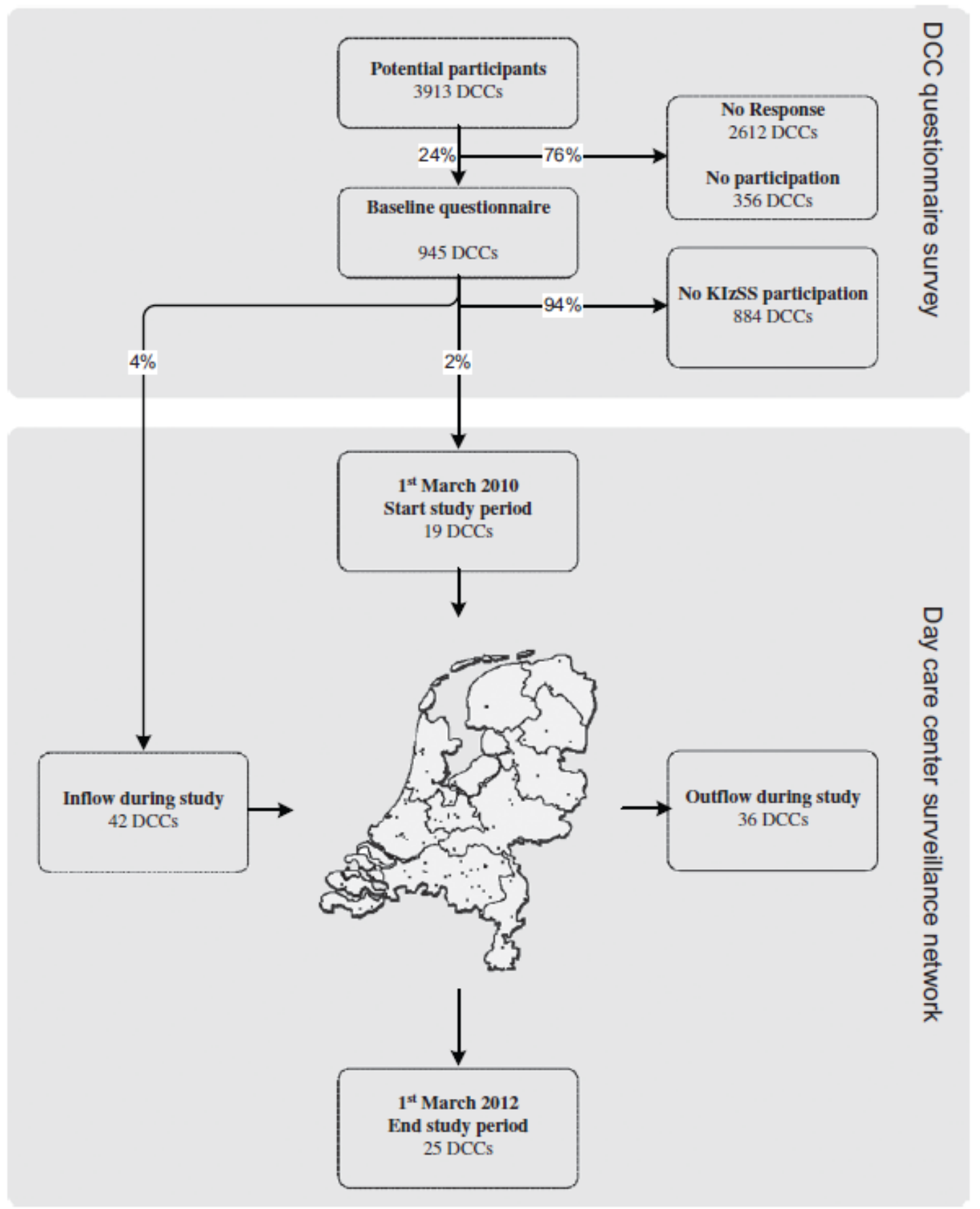


Enserink, R., Ypma, R., Donker, G.A., Smit, H.A., Pelt, W. van. Infectious disease burden related to child day care in the Netherlands. Pediatric Infectious Disease Journal: 2013, 32(8), e334e340

TABLE 2. Baseline Characteristics of DCCs That Participated in the November 2009 Questionnaire Survey $(n=945)$ and Differences Between DCCs That Participated $(n=40)$ and Never Participated $(n=905)$ in the Surveillance Network

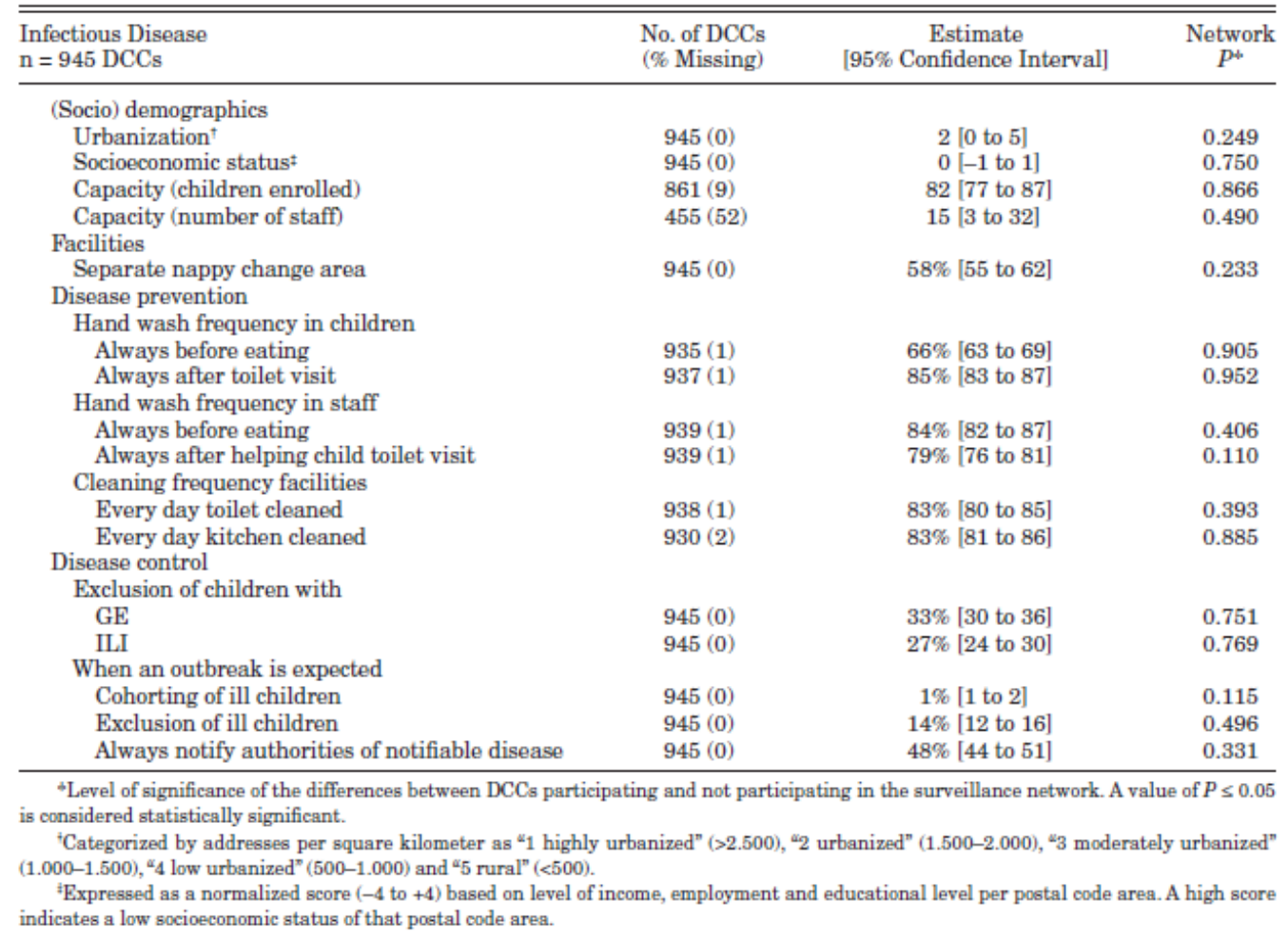


Enserink, R., Ypma, R., Donker, G.A., Smit, H.A., Pelt, W. van. Infectious disease burden related to child day care in the Netherlands. Pediatric Infectious Disease Journal: 2013, 32(8), e334= e340

FIGURE 2. Monthly infectious disease incidence per 1000 child-years of (A) GE; (B) ILI, ear infection and eye infection; and (C) exanthema, chicken pox and impetigo as measured in the KIzSS network from March 2010 to March 2012.
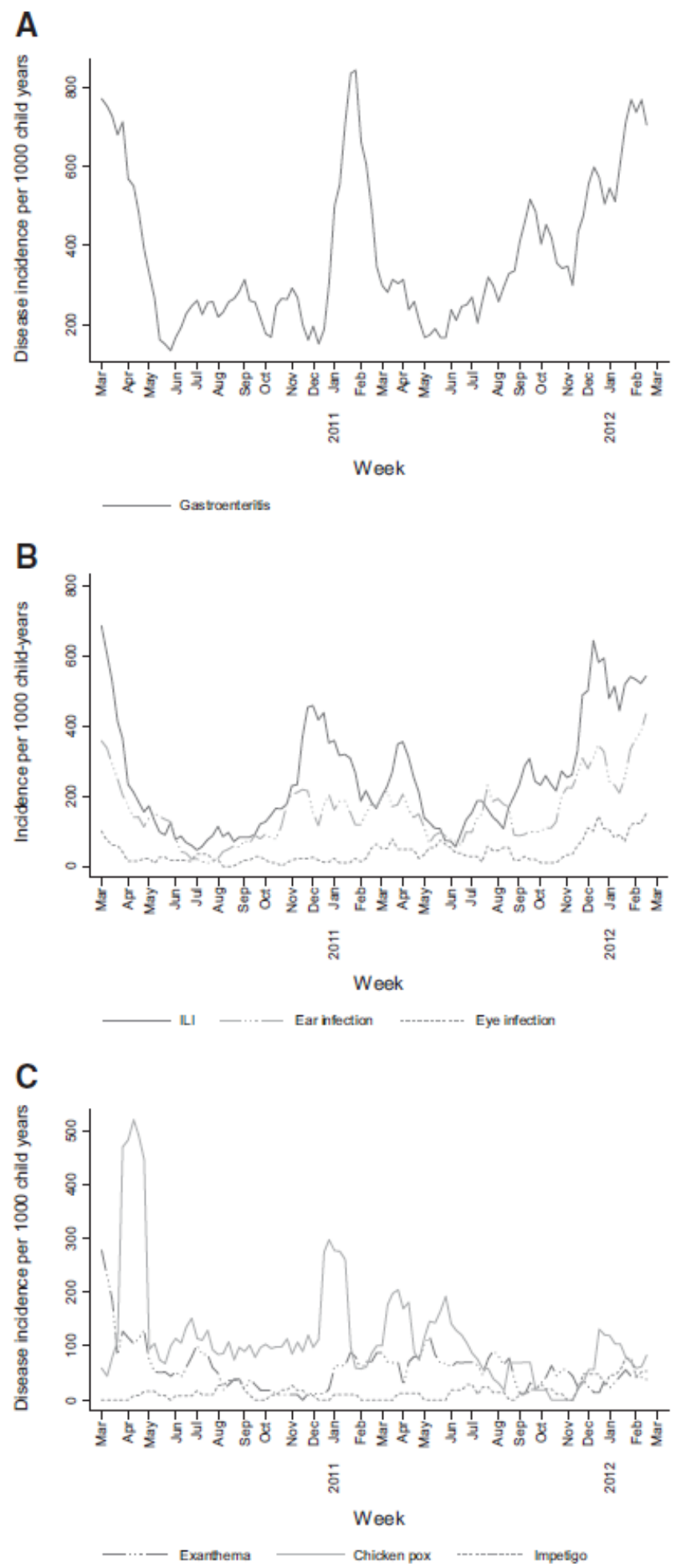
Enserink, R., Ypma, R., Donker, G.A., Smit, H.A., Pelt, W. van. Infectious disease burden related to child day care in the Netherlands. Pediatric Infectious Disease Journal: 2013, 32(8), e334e340

\begin{tabular}{|c|c|c|c|c|c|c|c|}
\hline $\begin{array}{l}\text { Episodes: } 2488 \\
\text { Child-years at } \\
\text { Risk: } 2441\end{array}$ & Episodes ${ }^{\circ}$ & Incidence* & $\begin{array}{l}\text { Study Year } \\
2010-2011 \text { vs. } \\
2011-2012\end{array}$ & $\begin{array}{c}\text { Age } \\
0-2 \text { years vs. } \\
2-4 \text { years }\end{array}$ & & Healthcar & Use \\
\hline $\begin{array}{l}\text { Infectious } \\
\text { Disease }\end{array}$ & $\mathrm{n}(\%)$ & $\begin{array}{c}\text { Episodes per } \\
1000 \text { Child-years } \\
\text { [95\% Confidence Interval] }\end{array}$ & $\mathrm{IRR}^{\dagger}$ & $\mathrm{IRR}^{ \pm}$ & $\begin{array}{c}\text { Antibiotics } \\
(\% \mathrm{n})\end{array}$ & $\begin{array}{l}\mathrm{GP} \\
(\mathscr{\%} \mathrm{n})\end{array}$ & $\begin{array}{c}\text { Hospitalization } \\
(\mathscr{\%} \mathrm{n})\end{array}$ \\
\hline GE & $944(38.0)$ & 387 [362-412] & $0.7[0.7-0.8]$ & $1.7[1.5-1.9]$ & $6(0.6)$ & $119(12.6)$ & $15(1.6)$ \\
\hline ILI & $603(24.2)$ & 247 [228-268] & $0.7[0.6-0.8]$ & $1.6[1.4-1.8]$ & $49(8.1)$ & $218(36.2)$ & $14(2.3)$ \\
\hline Ear infection & $367(14.8)$ & $150[135-167]$ & $0.7[0.6-0.8]$ & $2.1[1.9-2.4]$ & $78(21.3)$ & $226(61.6)$ & $16(4.4)$ \\
\hline Chicken pox & $294(11.8)$ & $120[107-135]$ & $1.5[1.3-1.7]$ & $1.2[1.0-1.4]$ & $1(0.3)$ & $26(8.8)$ & $2(0.7)$ \\
\hline Exanthema & $144(5.8)$ & $59[50-70]$ & $0.9[0.7-1.2]$ & $2.8[2.3-3.4]$ & $1(0.7)$ & $57(39.6)$ & $1(0.7)$ \\
\hline Eye infection & $94(3.8)$ & $39[31-47]$ & $0.4[0.2-0.5]$ & $3.6[2.8-4.5]$ & $4(4.3)$ & $27(28.7)$ & $0(0.0)$ \\
\hline Impetigo & $42(1.7)$ & 17 [12-23] & $0.5[0.3-0.8]$ & $0.5[0.3-0.9]$ & $10(23.8)$ & $39(92.9)$ & $0(0.0)$ \\
\hline Total & $2488(100)$ & 1019 [979-1060] & $0.8[0.7-0.8]$ & $1.7[1.6-1.8]$ & $149(6.0)$ & $712(28.6)$ & $48(1.9)$ \\
\hline
\end{tabular}

TABLE 4. Differences in GE and ILI Incidence Requiring a Visit to the General Practitioner (GP) Between Child DCC-based Surveillance and GP-based Surveillance Between March 2010 and March 2011 Stratified by Age

\begin{tabular}{lccc}
\hline \hline & \multicolumn{2}{c}{$\begin{array}{c}\text { Incidence per 1000 } \\
\text { Child-years }\end{array}$} & \\
\cline { 2 - 3 } Infectious Disease & DCC & GP & IRR \\
\cline { 2 - 3 } Requiring GP Visit & & & \\
\hline GE & $80[63-100]$ & $47[38-56]$ & $1.7[1.3-2.2]$ \\
$0-2$ yr & $36[26-48]$ & $19[14-26]$ & $1.9[1.4-2.6]$ \\
$2-4$ yr & $55[45-65]$ & $33[28-39]$ & $1.7[1.4-2.0]$ \\
Overall & & & \\
ILI & $157[133-184]$ & $70[59-81]$ & $2.3[1.9-2.7]$ \\
$0-2$ yr & $56[44-71]$ & $40[32-49]$ & $1.4[1.1-1.8]$ \\
$2-4$ yr & $101[88-115]$ & $55[49-63]$ & $1.8[1.6-2.1]$ \\
Overall & &
\end{tabular}

Expressed as an IRR. 